\begin{tabular}{c} 
International Journal of Engineering \& Technology, $7(2)(2018) 626-634$ \\
International Journal of Engineering \& Technology \\
SPC \\
Website: $\begin{array}{c}\text { www.sciencepubco.com/index.php/IJET } \\
\text { doi: } 10.14419 / \text { ijet.v7i2.9922 } \\
\text { Research paper }\end{array}$ \\
\hline
\end{tabular}

\title{
Face recognition based on stable uniform patterns
}

\author{
A. Mallikarjuna Reddy ${ }^{1 *}$, V. Venkata Krishna ${ }^{2}$, L. Sumalatha ${ }^{3}$ \\ ${ }^{1}$ Research Scholar, JNTU Kakinada, Asst. Professor, Anurag Group of Institutions (Autonomous), Hyderabad, India \\ ${ }^{2}$ Principal, Nishitha College of Engineering, Hyderabad, India \\ ${ }^{3}$ Professor, University College of Engineering, JNTUK, Kakinada, Andhra Pradesh, India \\ *Corresponding author E-mail: mallikarjunreddycse@cvsr.ac.in
}

\begin{abstract}
Face recognition (FR) is one of the challenging and active research fields of image processing, computer vision and biometrics with numerous proposed systems. We present a feature extraction method named "stable uniform local pattern (SULP)", a refined variant of ULBP operator, for robust face recognition. The SULP directly applied on gradient face images (in $\mathrm{x}$ and y directions) of a single image for capturing significant fundamental local texture patterns to build up a feature vector of a face image. Histogram sequences of SULP images of the two gradient images are finally concatenated to form the "stable uniform local pattern gradient (SULPG)" vector for the given image. The SULPG approach is experimented on Yale, ATT-ORL, FERET, CAS-PEAL and LFW face databases and the results are compared with the LBP model and various variants of LBP descriptor. The results indicate that the present descriptor is more powerful against a wide range of challenges, such as illumination, expression and pose variations and outperforms the state-of-the-art methods based on LBP.
\end{abstract}

Keywords: Expressions; Gradient; Histogram; Illumination; Pose; Robust; Uniform.

\section{Introduction}

Face recognition is one of the significant and challenging topics for decades in computer vision and pattern recognition and [1], [2]. This is due to its widespread practical applications, such as access control [3], smart card design [4], information security [5], surveillance system [6], expression cloning [7] and law enforcement follow-up [8]. Facial images captured under the real world environment may have expressions variations, dissimilar poses, varying illumination and occlusions such as sunglasses etc...These issues make the face recognition system as a challenging and interesting problem. Numerous FR methods are proposed in the literature to tackle the above challenges [9], [10].There are three most popular and dominant categories of face recognition systems i.e. the methods based on deep learning [11], holistic (global features) approaches[12], and face image descriptors (local feature or component based) [13], [14]. The well-known learning-based descriptors for face recognition are Local Quantized Patterns (LQP) [15], and Discriminate Face Descriptor (DFD) [16].The performance of these methods $[15,16]$ depends on unsupervised or supervised learning techniques. In the holistic method global features are extracted from the entire face image. The local feature based methods decomposes face image into local facial features. These local features represent the most discriminant intrinsic information of face image to build feature vector. The feature extraction methods should be robust against many challenges. The face image descriptor methods have several advantages like ease of use, data independence, and robustness to real-life challenges such as illumination and expression differences. The LBP [17] and Gabor wavelets [18] based methods are most well -known and popular methods for feature based face recognition. The local binary pattern (LBP) $[19,20]$ have aroused increasing interest among the researchers in image processing, pattern recognition and computer vision, due to its simplicity, tolerance to monotonic illumination changes and efficient representation of local structures of images. Researchers worked on LBP and derived facial features efficiently for different tasks, which include face recognition [21], [22], [23], face detection [24], facial expression analysis [26], [26], demographic (gender, race, age, etc.) classification [27]. Many variants of LBP have been proposed in the literature to improve its efficiency further and LBP based methods are combined with statistical methods. The other well-known variants of LBP include Transition LBP (TLBP) [28] and Direction coded LBP (DLBP) [28].The methods based on Gabor wavelets [29] are proposed in the recent literature, to obtain more precisely multiscale and multi-orientation information of face images. A method for recognition on blurred faces [30] and another method based on edge magnitudes [31] are also proposed in the literature.

The existing face image descriptors, achieved successful performance but at the cost of high dimensionality and computational cost. Therefore there is a necessity to derive a face descriptor with superior performance with lower computational cost and feature size. To address this, in this work, we propose a novel feature extraction method called as Stable Uniform Local Pattern of gradients (SULPG) forFR. This descriptor provides an integrated way to capture stable uniform local patterns from two gradient images corresponding to a single image.

The remainder of the paper is organized as follows: Section 2 details the proposed SULPG descriptor. Experimental results are presented in Section 3, leading to conclusions in Section 4.

\section{Formation of stable uniform local pattern (SULP)}

An image is composed of micro patterns. The LBP can be viewed as first-order circular derivative patterns. The derivation of LBP 
code is a "two-step" process. In the initial step, each neighboring pixel of a " $3 \times 3$ neighborhood", is compared with its central pixel and binary patterns are derived using equation 1.In the second step, a unique decimal number, which is called as LBP or LBP code, is derived based on equation 2 . The LBP code holds significant information about the distribution of edges and other local features in an image.The neighboring pixels are denoted by $\mathrm{P}=$ $\left\{\mathrm{p}_{0}, \mathrm{p}_{1} \ldots \mathrm{p}_{7}\right\}$, the central pixel by $\mathrm{p}_{\mathrm{c}}$ and $\mathrm{R}$ represents the radius of the neighborhood.

$\mathrm{b}_{\mathrm{i}}= \begin{cases}1 & \Delta \mathrm{p}_{\mathrm{i}} \geq 0 \\ 0 & \Delta \mathrm{p}_{\mathrm{i}}<0\end{cases}$

Where $\Delta \mathrm{p}_{\mathrm{i}}=\mathrm{p}_{\mathrm{i}}-\mathrm{p}_{\mathrm{c}}$

For each $3 \times 3$ neighborhood, a unique LBP code is derived from the equation (2)

$\mathrm{LBP}_{\mathrm{P}, \mathrm{R}}=\sum_{\mathrm{i}=0}^{\mathrm{i}=7} \mathrm{~b}_{\mathrm{i}} * 2^{\mathrm{i}}$

The $\mathrm{LBP}_{\mathrm{P}, \mathrm{R}}$ operator produces $2^{\mathrm{P}}$ different output values.

The basic LBP operator was later derived for different number of neighboring pixels ' $\mathrm{P}$ ' and radius ' $\mathrm{R}$ ' . The uniform patterns are introduced by manenpaa et al. [32]. It is observed that more than $90 \%$ of the windows in human faces and in natural textures are uniform LBPs (ULBP). This proposition was further confirmed by ojala et al. [19]. The commonality in uniform LBP (ULBPs) is that they contain at most two, "zero to one or one to zero transitions" in the circular binary code. The uniformity of ' $U$ ' of a neighborhood $\mathrm{G}$, is measured in the following way

$\mathrm{U}(\mathrm{G})=\operatorname{abs}\left(\mathrm{s}\left(\mathrm{g}_{\mathrm{p}-1}-\mathrm{g}_{\mathrm{c}}\right)-\mathrm{S}\left(\mathrm{g}_{\mathrm{o}}-\mathrm{g}_{\mathrm{c}}\right)+\Sigma \mathrm{S}\left(\mathrm{g}_{\mathrm{p}}-\mathrm{g}_{\mathrm{c}}\right)-\mathrm{S}\left(\mathrm{g}_{\mathrm{p}-1}-\mathrm{g}_{\mathrm{c}}\right)\right.$

The neighborhood with $U(G)$ is less than or equal to two are termed as uniform local binary pattern (ULBP).

The total number of uniform patterns on a circular neighborhood of $(\mathrm{P}, \mathrm{R})$ is $\mathrm{P}+(\mathrm{P}-1)+2$. The remaining $2^{\mathrm{P}}-(\mathrm{P}+(\mathrm{P}-1)+2)$ codes are termed as non-ULBP (NULBP) codes. However, it is noted that at most $10 \%$ of the windows in the texture images will have NULBP windows. It is observed that NULBPs generate noise and high dimensionality. Therefore the NULBPs are represented under one label and treated them as miscellaneous. Allocating one label for all large number of NULBPs drastically reduces overall dimensionality and also overcomes the problem of noisy patterns There are 58 ULBPs and 198 NULBPs on a $3 \times 3$ window with $\mathrm{P}=8$ and $\mathrm{R}=1$. In this case, the dimensionality of the histogram by considering the LBP and ULBP will be 256 and 59 (in this case all NULBPS are treated under one label). The present paper after studying the ULBPs trend on facial images derived stable uniform local patterns (SULP). The SULP is a subset of ULBP that consists of more than $92 \%$ of ULBPs. The SULPs can be treated as the dominant ULBPs in general. The SULP is defined as: "The SULP is a ULBP that contains"

1) All zero transitions of ULBP.

2) All transitions that occur from two or more adjacent ones to two or more adjacent zeros or vice versa in a circular manner. These transitions are more stable transitions.

For example, the LBP code 239 i.e. 11101111 is a ULBP, however it is not stable in terms of human perception because a single zero (low intensity) in between all high intensity values, is difficult to recognize and in most of the cases, it is recognized as a complete blob i.e. high intensity. The LBP code 205 i.e., 11001111 is a ULBP and also a SULP, because "the human perception in recognizing, two or more zeros in between remaining high intensity values", will be very high when compared to the above case of LBP code 239. The SULP considered the zero transitions because it represents both blobs and complete holes and further they are more stable. There are exactly two ULBPs that will have zero transitions: all zeros and all ones. There are exactly 46 ULBPs that represent the second type of transitions i.e. the transitions that occur after two or more consecutive ones followed by two or more consecutive zeros on the circular neighborhood.
The total number of "stable uniform local patterns (SULP)" on a circular neighborhood with $(\mathrm{p}, \mathrm{R})$ is $\mathrm{p} *(\mathrm{p}-2)$. This drastically reduces the dimension and preserves the fundamental properties of texture, which is sufficient for face recognition.

The present paper computed the percentages of ULBPs, SULPs of different databases and are listed in Table 1 . From table 1 it is evident that SULP occupies on average, $88 \%$ of windows in the raw facial images and $93.23 \%$ of ULBP windows.

Table 1: Percentage of SULP Windows on Different Facial Databases with $\mathrm{P}=8$ and $\mathrm{R}=1$

\begin{tabular}{llll}
\hline $\begin{array}{l}\text { Facial Data- } \\
\text { base }\end{array}$ & $\begin{array}{l}\text { \% ULBP In raw } \\
\text { image }\end{array}$ & $\begin{array}{l}\text { \% SULP In raw } \\
\text { image }\end{array}$ & $\begin{array}{l}\text { \% SULP in } \\
\text { ULBP }\end{array}$ \\
\hline Yale & 96.67 & 91.57 & 94.77 \\
ATT & 97.10 & 92.31 & 95.06 \\
FERET & 92.77 & 86.96 & 90.57 \\
CAS-PEAL & 93.42 & 90.15 & 91.45 \\
LFW & 92.45 & 86.44 & 92.56 \\
Average & 94.74 & 89.48 & 93.23 \\
\hline
\end{tabular}

\subsection{Derivation of stable uniform local pattern (SULPG)}

The human visual system is more sensitive to local gradient changes of intensity, than the image intensity itself $[33,34]$. The present SULPG descriptor, extracts features on gradient images instead of raw images. Let I is an image and $I(x, y)$ represents the intensity value of the pixel location (x,y) of the image I. The present SULPG descriptor derives initially two directional gradient images along $\mathrm{x}$-axis and $\mathrm{y}$-axis $(\mathrm{Gx}, \mathrm{Gy})$ from the input image $\mathrm{I}$, using the equations 4 and 5 .

$\mathrm{Gx}=1 / 2 \cdot \mathrm{I}(\mathrm{x}+1, \mathrm{y})-(1 / 2 \cdot \mathrm{I}(\mathrm{x}-1, \mathrm{y}))$

$\mathrm{Gy}=1 / 2 \cdot \mathrm{I}(\mathrm{x}, \mathrm{y}+1)-(1 / 2 \cdot \mathrm{I}(\mathrm{x}, \mathrm{y}-1))$

This paper gained advantage in deriving efficient feature vectors from facial images by initially computing gradient images because gradients are proved to be more sensitive of visual human system than local intensity differences. Extraction of texture information from gradient images is more efficient than from raw image [33], [34].Then, on these directional gradient images the present paper derives SULP feature vectors to extract stable uniform local patterns. The stable Uniform local patterns which are binary strings derived based on bitwise transitions from 0 to 1 or 1 to 0 , in a circular manner are utilized to reduce the SULPG descriptions' length. The SULP histogram sequences of these two gradient images (SULP-G ${ }_{x}$ and SULP-Gyof a single image) are concatenated to derive SULPG description of the image. This makes the feature size (number of histogram bins) of SULPG as $2 \times 43=86$.

\section{Results and discussions}

In this section, the performance of SULPG descriptor is computed. To test the advantages of SULPG, the performance of seven stateof-the-art face image descriptors, i.e., LBP [20], LTP [22], ULBP[32], LPQ [30], PLBP [35], multi-scale LBP (MsLBP) [19], Multi-scale DLBP (MsDLBP) [36], are also presented. These existing descriptors [19], [20], [22], [30], [32] are easy to implement and they are based on local features. The cropped face image data for these descriptors in the present paper are exactly the same. For efficient face recognition the present paper evaluated histograms of LBP, ULBP, LTP, MPLBP and SPLBP on each individual facial image and placed in training database. In the similar way the above histograms are evaluated for test facial image and the face recognition is evaluated based on Chi-square distance metric as given in equation 6 .

$\mathrm{R}(\mathrm{d}, \mathrm{t})=\min \left(\sum_{\mathrm{i}=1}^{\mathrm{n}}\left(\left(\mathrm{d}_{\mathrm{i}}-\mathrm{t}_{\mathrm{i}}\right)^{2} /\left(\mathrm{d}_{\mathrm{i}}+\mathrm{t}_{\mathrm{i}}\right)\right) / 2\right)$

Where $\mathrm{d}, \mathrm{t}$ are two image features (histogram vectors) and $\mathrm{R}(\mathrm{d}, \mathrm{t})$ is the histogram distance for recognition. 
For experimental sake this paper used the most popular and widely used facial databases .i.e., FERET (face recognition technology) [37], CAS-PEAL [38], LFW (Labeled Faces in the Wild) [39]; Yale Face Database B [40]; AT\&T ORL [41].

FERET database contains two sub categories of databases namely, Frontal FERET Image Sets and Non-Frontal FERET Image Sets The frontal image sets i.e. $\mathrm{F}_{\mathrm{a}}, \mathrm{F}_{\mathrm{b}}, \mathrm{F}_{\mathrm{c}}$, Duplicate I (Dup I) and Duplicate II (Dup II) are part of the Frontal FERET Image Set. There $F_{a}$ set, images are used for gallery and training sets. The $F_{b}, F_{c}$, Dup I and Dup II image sets are used for probe sets and they consist of 1195, 194, 722 and 234 images respectively. Apart from frontal images, FERET has pose-view images of 200 people, which are Non-Frontal FERET Image Sets. This paper considered only the frontal FERET data sets for our experimental purpose.

The CAS-PEAL face database includes Chinese face database with different sources of variations, especially Pose, Expression, Accessories, and Lighting (PEAL). Currently, the CAS-PEAL face database contains 99,594 images of 1040 individuals (595 males and 445 females) with varying Pose, Expression, Accessory, and Lighting (PEAL). A subset of the whole CAS-PEAL face database referred as CAS-PEAL-R1, with 30,900 images of 1040 subjects is publicly available now. The CAS-PEAL-R1 images are divided in to two categories: the frontal and non-frontal. This database contains one training set, one gallery set, and six probe sets in frontal category and three in non-frontal category. The six probe sets of frontal category are denoted as PE, PA, PL, PT, PB, and PS correspond to variations in expression, accessory, lighting, time, background, and the distance of frontal faces, respectively. The three categories of non-frontal probe sets PU, PM, and PD correspond to one type of pose variation i.e. looking: upward, towards camera and downwards.

The LFW [39], face photographs are designed for studying the problem of unconstrained face recognition. The data set contains more than 13,000 images of faces organized in to two views: view1 and view 2 .LFW is a challenging dataset since its images contain large pose, expression, and illumination variations. For model identification and parameter tuning the view 1 set of LFW is used. For performance reporting View 2 is used. In View 2 two models are used to make use of the training set: image-restricted paradigm and unrestricted paradigm. In the first model the training is made on officially defined images. In the second model the identity information of the training images can be used. For model identification and parameter tuning the view 1 set of LFW is used. There are 5760 images of 10 subjects are available in the Yale Face Database B. These images are derived from 576 viewing conditions (9 poses x 64 illumination conditions).

The sample images of these databases are shown in Fig. 1, 2, 3 and 4. The SULP-G $\mathrm{G}_{\mathrm{x}}$ and SULP-G $\mathrm{G}_{\mathrm{y}}$ facial images of the present descriptor are shown in Fig. 5, 6, 7 and 8 for facial data bases YALE, FERET, CAS-PEAL and LFW respectively.

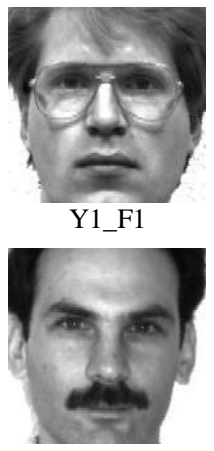

Y2_F1

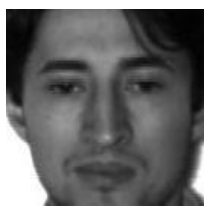

Y3_F1

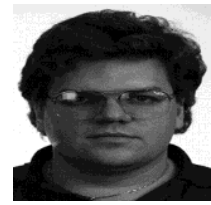

4

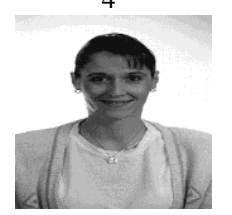

34

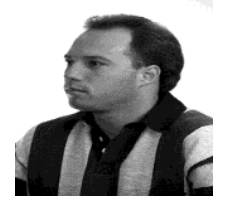

22

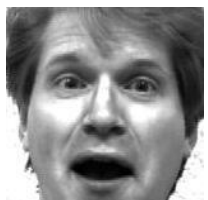

Y1_1F2

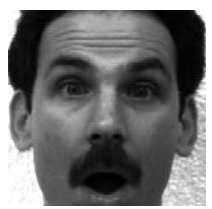

Y2_F2

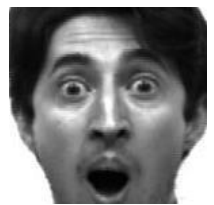

Y3_F2
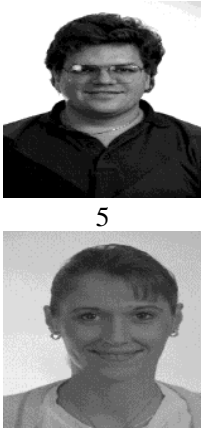

35

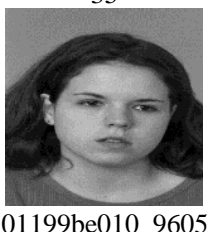

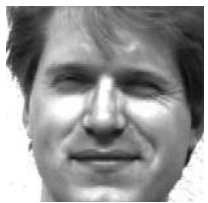

Y1_F3

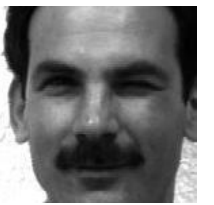

Y2_F3

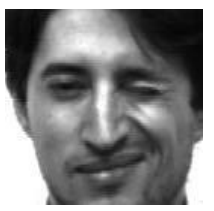

Y3_F3

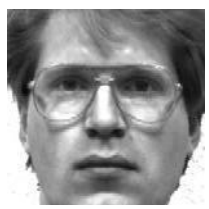

Y1_F4

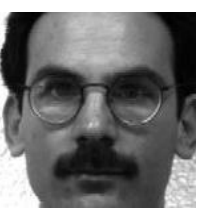

Y2_F4

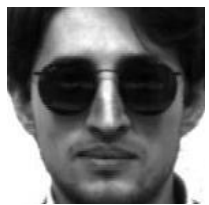

Y3_F4

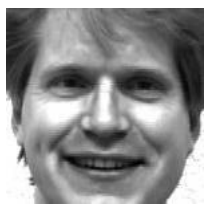

Y1_F5

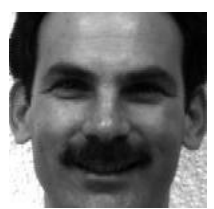

Y2_F5

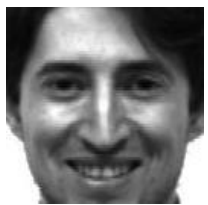

Y3_F5

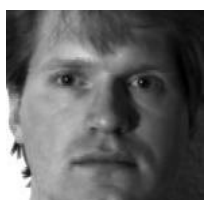

Y1_F6

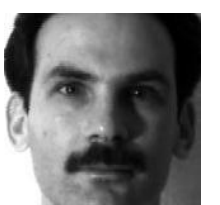

Y2_F6

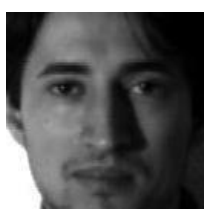

Y3_F6

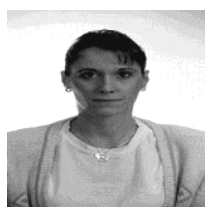

32

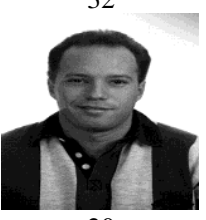

20

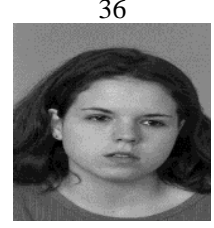

01199be010_9605

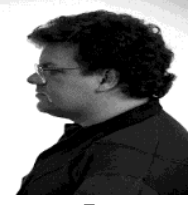

7

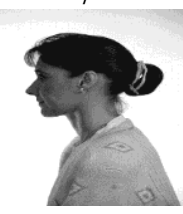

37

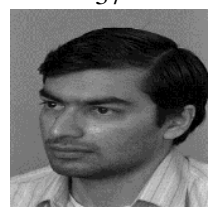

01206bh010 9605
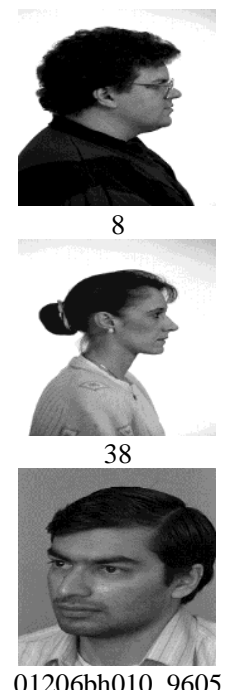

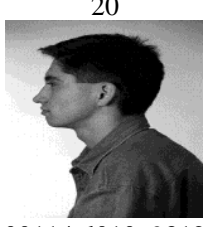

00114p1010_9312

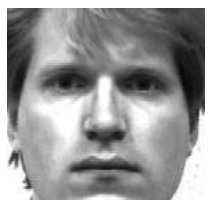

Y1_F7

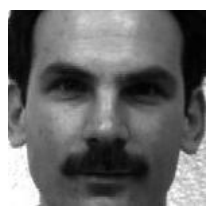

Y2_F7

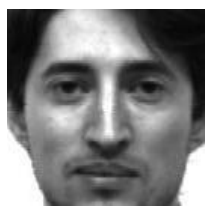

Y3_7

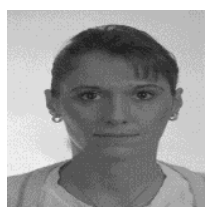

33

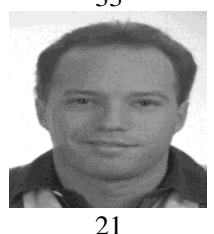

21

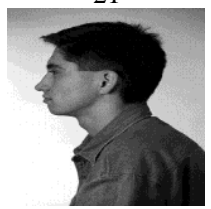

00114p1010 9312

Fig. 2: Sample Facial Images of FERET Database. 

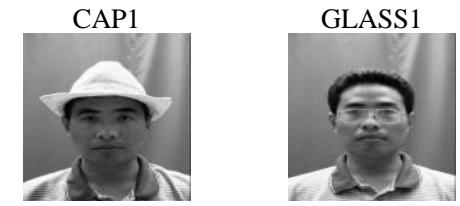

SMILE

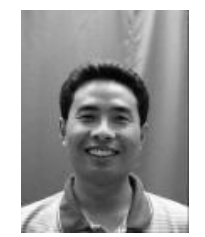

FY 000025 IEU+0 0_PM+00_EN_A0_ D1_T0_BB_M0_R 0_S0
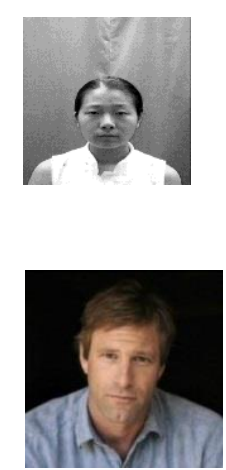

Aa-

ron_Eckhart_0001

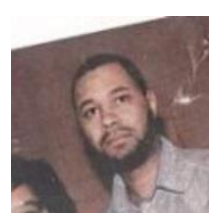

Aa-

ron_Patterson_0001

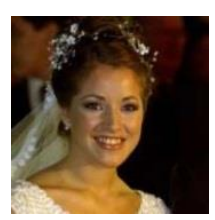

Giselle_Estefania_T avarelli_0001

OPENMOUTH

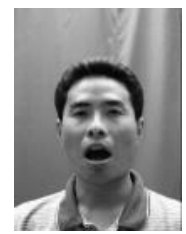
0_S0

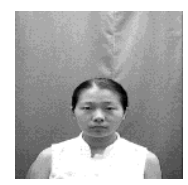

Aa-

Aa-

001

Giu-
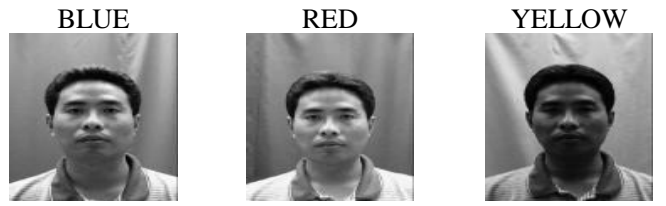

LIGHT1

FY 000025_IEU+0 0_PM+00_EN_A0_ D2_T0_BB_M0_R

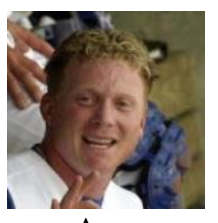
ron_Guiel_00

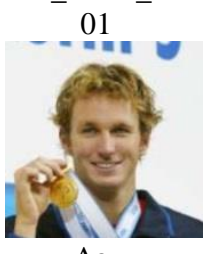

ron_Peirsol_0

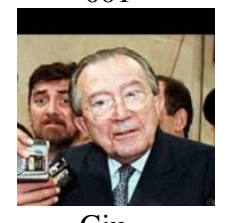

lio_Andreotti

_0001

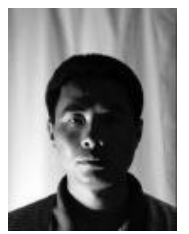

FY 000229 IEU+0 0_PM+00_EN_A1 D0_T0_BB_M0_R 1_S0

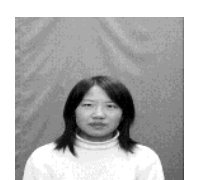

Fig. 3: Sample facial images of CAS-PEAL database.

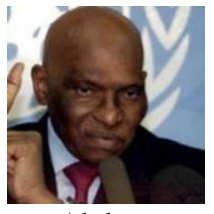

Abdoulaye_Wade 0004

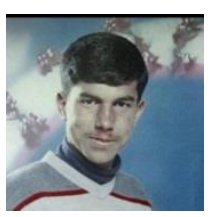

$\mathrm{Ab}-$

del_Madi_Shabn eh_0001

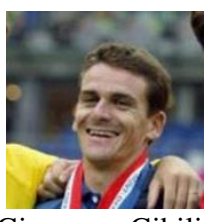

Giuseppe_Gibilis co_0003

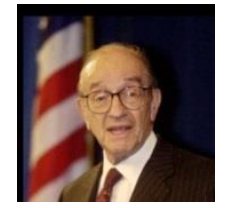

Alan_Greenspa n_0003

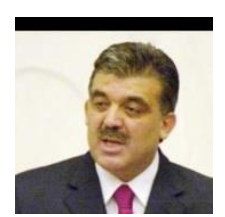

Abdullah_Gul_0001

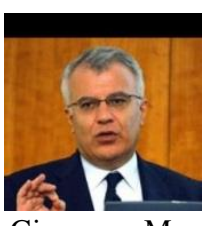

Giuseppe_Mor chio_0001

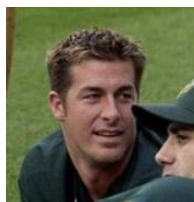

Mark_Mulde r_0001

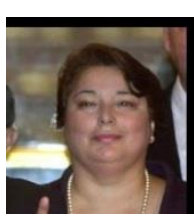

Beatriz Merino

0001

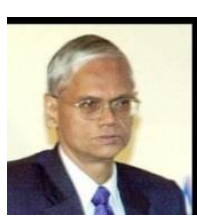

GL Peiris 0

003

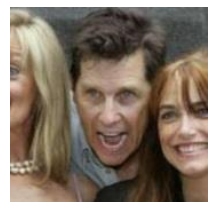

Tim_Matheson 0001

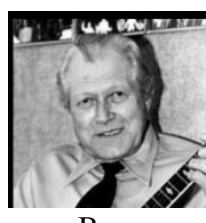

Bee-

cher_Ray_Kirb y_0001

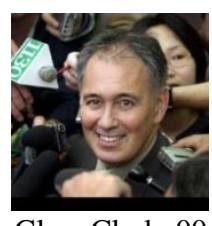

Glen_Clark_00

01

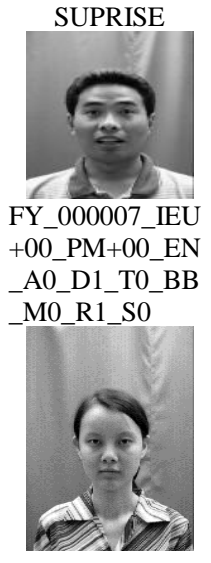

FY 000438_IEU +00_PM+00_EN _A0_D0_T0_BR _M0_R1_S0

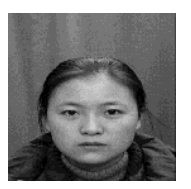

Fig. 4: Sample Facial Images of LFW Face Database.

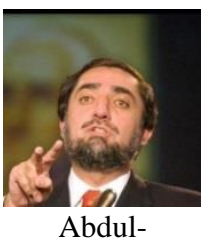

lah_Ahmad_Badaw i_0001

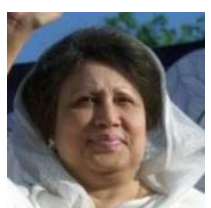

Begum_Khaleda_Zi a_0001

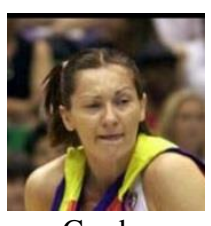

Gordana_Grubin_0001 

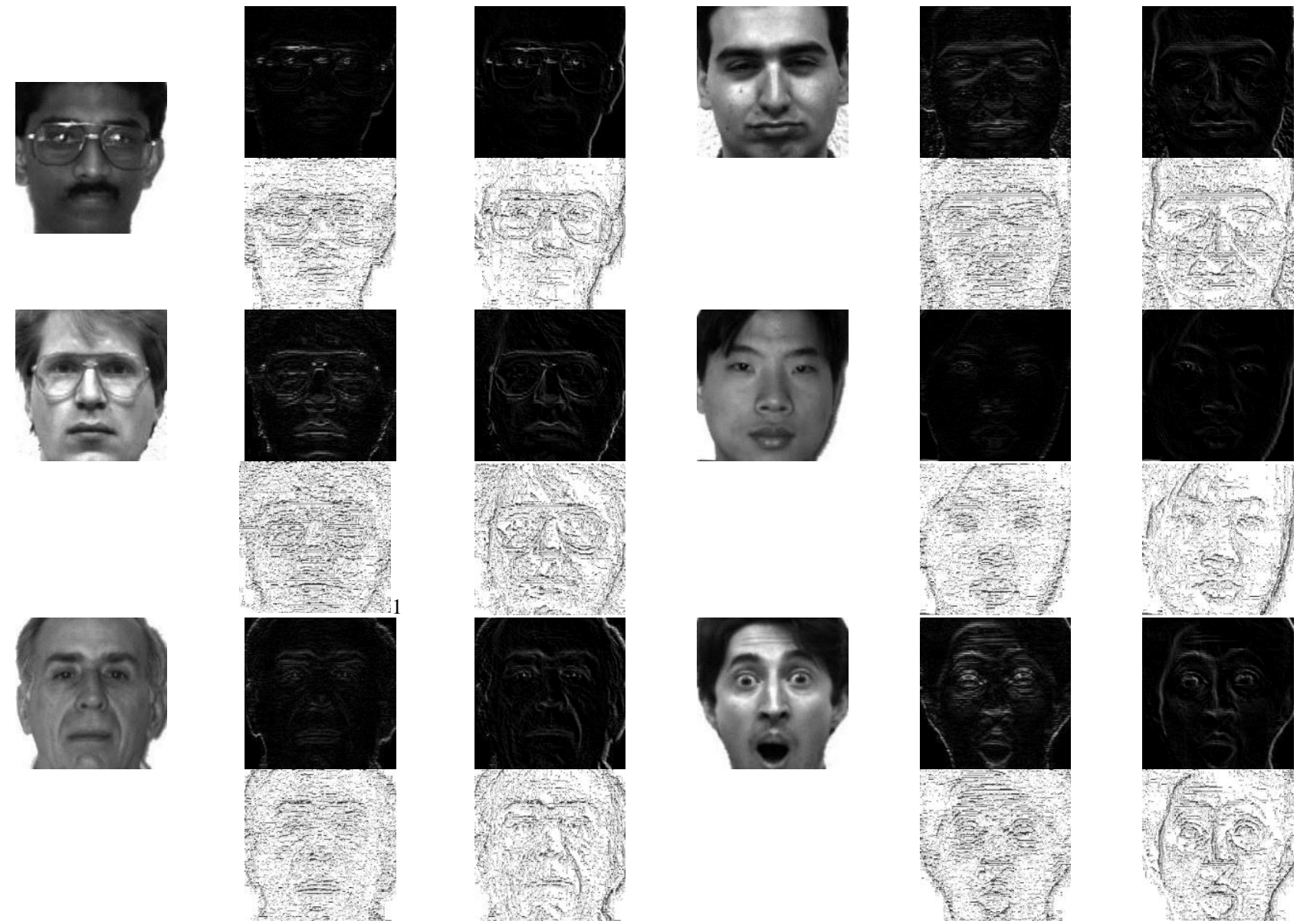

Fig. 5: (A) Original YALE Image (B) X-Gradient Image (C) Y-Gradient Image (D) SULP-Gximage (E) SULP-Gyimage
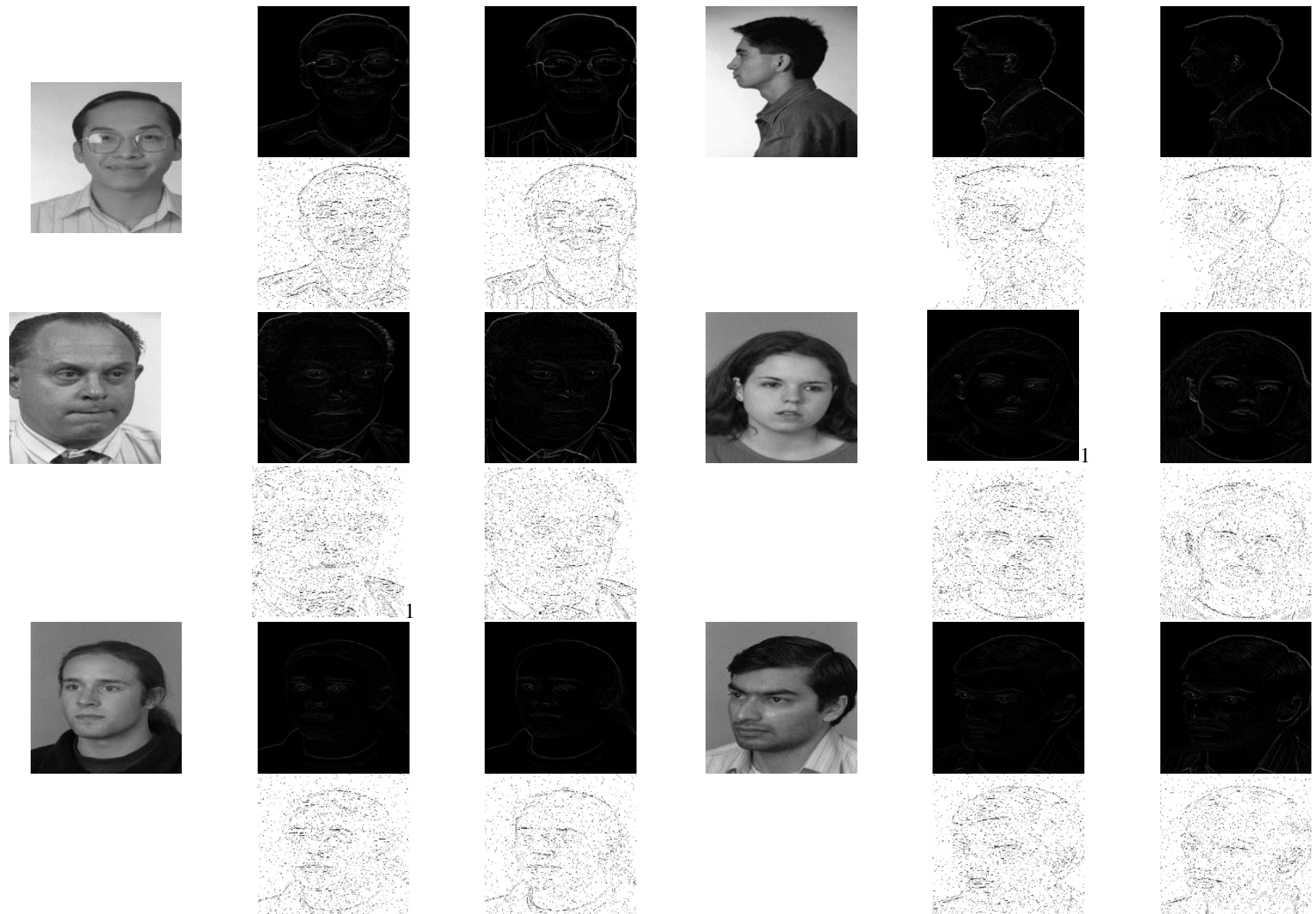

Fig. 6: (A) Original FERET Image (B) X-Gradient Image (C) Y-Gradient Image (D) SULP-Gximage (E) SULP-Gyimage. 

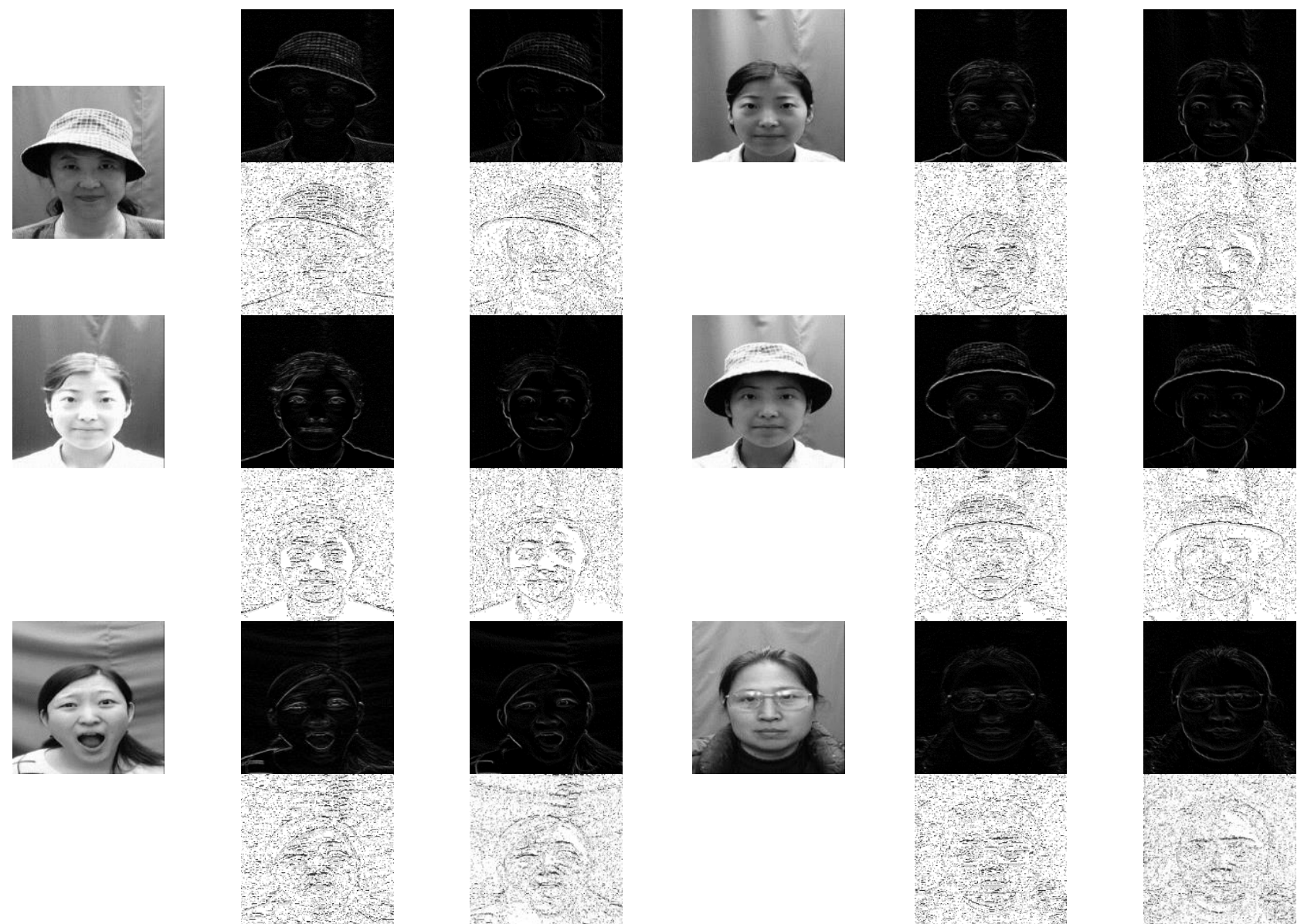

Fig. 7: (A) Original CAS-PEAL Image (B) X-Gradient Image (C) Y-Gradient Image (D) SULP-Gximage (E) SULP-Gyimage
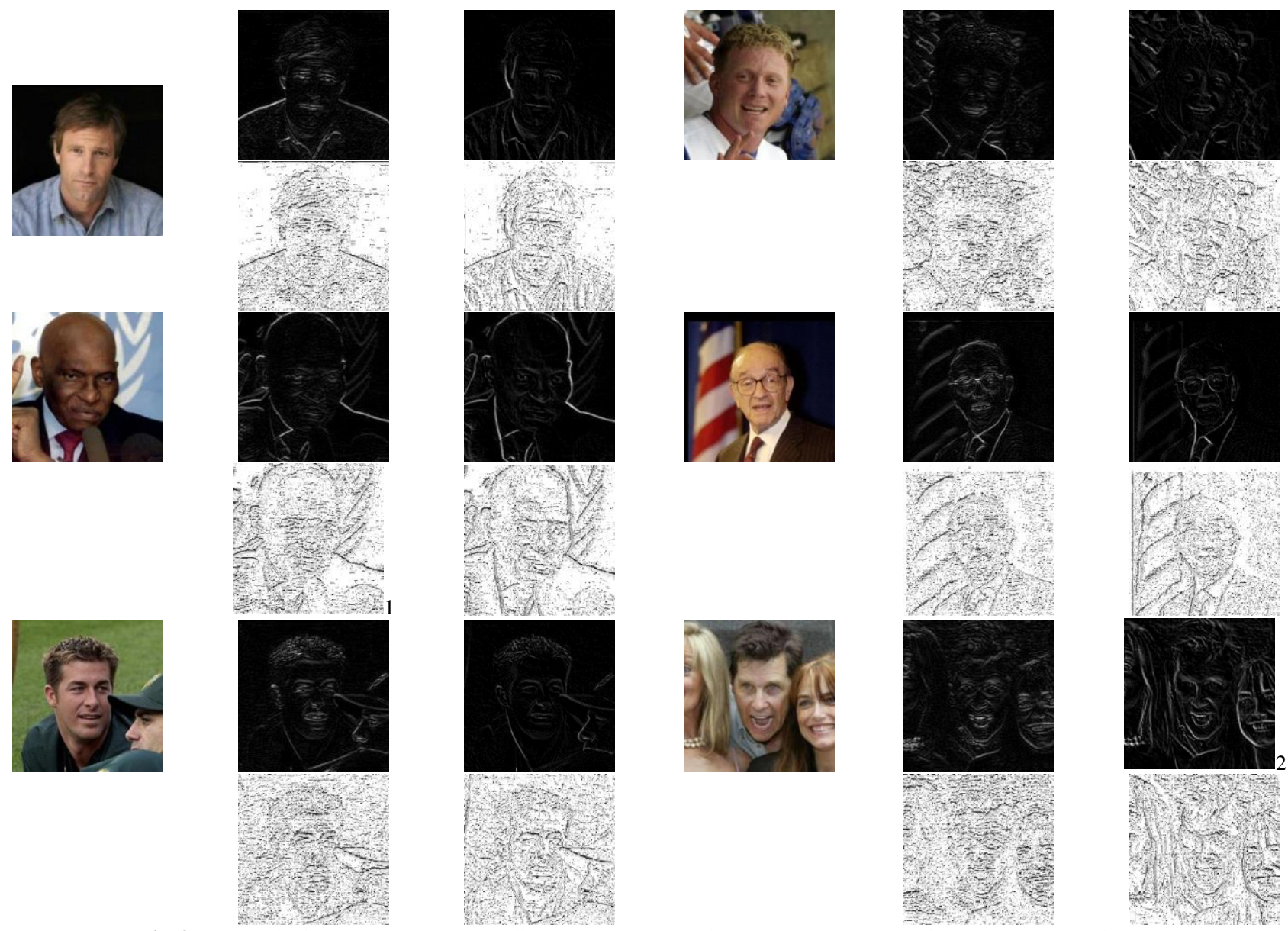

Fig. 8: (A) Original LFW Image (B) X-Gradient Image (C) Y-Gradient Image (D) SULP-Gximage (E) SULP-Gyimage. 
The Fig. 5 to Fig. 8 demonstrates the advantages of gradient approach. The enhanced edge information is an essential feature to represent a facial images. This is achieved in the present paper by computing SULP a gradient images (Fig 5 to 8). The illumination condition effect on SULP gradient images is less than raw intensity ones, and thus feature extraction will be more efficient. The proposed SULPG approach is different from many other intensity based methods as well as gradient magnitudes and gradient orientation based ones ([23], [42], [43].

The SULPG descriptor also enriches discrimination power with meaningful visual features i.e. local contrast, edges, discontinuities properties, (from gradient images), thus making SULPG more robust against face recognition the challenges like illumination, pose and time-lapse variations.

Table 2: Identification Rates for Different Descriptors on FERET.

\begin{tabular}{lllllll}
\hline & $\mathrm{Fb}$ & $\mathrm{Fc}$ & Mean $(\mathrm{Fb}$ and $\mathrm{FC})$ & $\mathrm{Dup} 1$ & Dup2 & Mean of DUP1 and DUP2 \\
\hline LBP & 96.90 & 98.45 & 97.68 & 83.93 & 90.44 & 87.19 \\
LTP & 96.90 & 98.97 & 97.94 & 83.93 & 83.76 & 83.85 \\
LPQ & 97.41 & 99.48 & 98.45 & 82.69 & 81.62 & 82.16 \\
ULBP & 96.02 & 97.92 & 96.97 & 82.90 & 88.24 & 85.57 \\
PLBP & 97.00 & 98.01 & 97.51 & 83.28 & 82.90 & 83.09 \\
MsLBP & 97.07 & 98.97 & 98.02 & 83.38 & 83.33 & 83.36 \\
MsDLBP & 95.65 & 97.94 & 96.8 & 79.09 & 79.09 & 79.09 \\
SULPG & 97.50 & 98.50 & 98.0 & 84.20 & 83.90 & 80.55 \\
\hline
\end{tabular}

Table 3: Rank-1 Identification Rates for Different Face Image Descriptors on the Nine Probe Sets of PEAL

\begin{tabular}{|c|c|c|c|c|c|c|c|c|c|c|c|c|}
\hline Descriptors & $\mathrm{PE}$ & PA & PT & PB & PS & PL & PU & PM & PD & $\begin{array}{l}\text { Mean } \\
\text { (PE-Ps) }\end{array}$ & $\begin{array}{l}\text { Mean(PE- } \\
\text { PL) }\end{array}$ & $\begin{array}{l}\text { Mean } \\
\text { (PU- } \\
\text { PD) }\end{array}$ \\
\hline LBP & 94.27 & 91.82 & 100.0 & 99.46 & 99.64 & 46.90 & 60.32 & 83.66 & 44.60 & 97.04 & 88.68 & 62.86 \\
\hline LTP & 94.39 & 91.77 & 100.0 & 99.46 & 99.64 & 47.17 & 61.32 & 84.46 & 44.68 & 97.05 & 88.74 & 63.49 \\
\hline LPQ & 93.95 & 92.39 & 100.0 & 99.28 & 99.27 & 57.16 & 57.78 & 86.46 & 44.76 & 96.98 & 90.34 & 63.00 \\
\hline ULBP & 94.07 & 91.92 & 100.0 & 99.28 & 99.48 & 45.24 & 58.28 & 82.78 & 43.92 & 96.95 & 88.33 & 61.66 \\
\hline PLBP & 94.87 & 92.02 & 100.0 & 99.86 & 99.84 & 46.02 & 60.01 & 82.94 & 44.28 & 97.32 & 88.77 & 62.41 \\
\hline MsLBP & 95.16 & 92.04 & 100.0 & 99.46 & 99.64 & 47.75 & 61.76 & 85.16 & 44.88 & 97.26 & 89.01 & 63.93 \\
\hline MsDLBP & 92.42 & 90.63 & 100.0 & 99.28 & 99.27 & 48.11 & 55.00 & 82.03 & 37.03 & 96.32 & 88.29 & 58.02 \\
\hline SULPG & 95.46 & 93.72 & 100.0 & 99.48 & 99.62 & 52.4 & 61.89 & 89.12 & 47.02 & 97.66 & 90.11 & 66.01 \\
\hline
\end{tabular}

The FR rate results on FERET frontal datasets are recorded in Table 3 and the following observations are made:

1) High performance is recorded by all descriptors on $\mathrm{Fb}$ and Fc probe sets when compared to DUP1 and DUP2 probe sets. This is due to the well-controlled environment of $\mathrm{Fb}$ and FC probe sets. The SULP achieves more than $1 \%$ of high mean classification rate when compared to other descriptors. The descriptors exhibited a little high recognition rate on probe set $\mathrm{Fc}$ when compared to $\mathrm{Fb}$.

2) There is a considerable drop in the performance of DUP1 and DUP2 probe sets by all descriptors. This is because the images in DUP1 and DUP2 exhibit expression and illumination variation when compared to $\mathrm{Fb}$ and $\mathrm{Fc}$.

3) The proposed SULPG has shown high recognition rate when compared to other descriptors at low dimensionality.

4) The present paper also computed the overall mean recognition rate for $\mathrm{Fa}$ and $\mathrm{Fb}$ probe sets and also for DUP1 and DUP2 probe sets and listed Table 2.

For each descriptor the present paper computed FR rate using nine probe sets of CAS-PEAL-R1, which includes both frontal and non-frontal subsets and listed in Table 3 . The following observations are noted:

1) All descriptors have shown high FR rate on frontal probe subsets i.e. PE, PA, PT, PB, PS. The proposed SULPG resulted with the high FR rate on all these five probe sets

2) All descriptors except LPQ have shown a poor FR rate around $45 \%$ on the PL probe set. This is mainly because of high illumination and image blur variation of PL set. The LPQ could sustain from these variations and resulted a $10 \%$ of the high FR rate i.e. around 57\%.

3) We have also computed the mean recognition rate of frontal subsets (excluding and also including PL) and listed in the Table 3. The mean FR rate of SULP is higher than the rest of the descriptors with low dimensionality except LPQ.

4) On non-frontal probe sets, the probe set PM has exhibited high performance due to the pose of the face with respect to camera. After PM the probe set PU attained better performance than PD because capturing of facial information will be more in this pose (PU) when compared to PD.
5) The mean FR rate clearly exhibits the superiority of the present descriptor and robustness to pose variations on probe sets PM, PD,PU over the rest of the descriptors

The recognition rate on LFW dataset is reported in Table 4 and the following are note down.

Table 4: Recognition Rate on LFW Database

\begin{tabular}{lc}
\hline & TFW \\
\hline Descriptors & 72.43 \\
\hline LBP & 72.65 \\
LTP & 73.27 \\
ULBP & 72.68 \\
LPQ & 72.78 \\
PLBP & 72.88 \\
MsLBP & 72.17 \\
MsDLBP & 73.54 \\
SULPG
\end{tabular}

1) All the descriptors achieved the FR rate around $75 \%$ for LFW data set and this is due to large variations in pose, expression, and illumination in LFW.

2) The proposed SULPG achieved superior performance on LFW dataset when compared to other descriptors.

The FR rates of Yale face database B are listed in the Table 5 and the following are noted.

1) All the descriptors has shown high FR rate and this may be due to small dataset.

2) The LPQ have exhibited a high recognition rate because LPQ is viewed as illumination invariant.

Table 5: Recognition Rate on the YALE-B Database

\begin{tabular}{ll}
\hline Descriptors & YALE \\
\hline LBP & 81.94 \\
LTP & 82.01 \\
ULBP & 83.61 \\
LPQ & 82.43 \\
PLBP & 83.06 \\
MsLBP & 83.86 \\
MsDLBP & 81.91 \\
SULPG & 85.78 \\
\hline
\end{tabular}


The present paper computed the feature vector size the (number of histogram bins or dimension) for each descriptor and listed Table 6.

Table 6: Feature Sizes of the Proposed and Existing Facial Image Descriptors

\begin{tabular}{llll}
\hline Descriptor & Feature size & Descriptor & Feature size \\
\hline LBP[20] & 256 & PLBP[35] & 144 \\
LTP[22] & 512 & MsLBP[19] & 512 \\
ULBP[19] & 59 & MsDLBP[36] & 512 \\
LPQ[30] & 256 & Proposed-SULPG & $86(2 \times 43)$ \\
PULBP & 96 & & \\
\hline
\end{tabular}

The Table 8 clearly demonstrates that the feature vector dimensions of LBP and LPQ are almost three times higher than SUPLG, whereas the LTP, MsLBP and MsDLBP feature vectors are six times higher than SULPG. Finally, from this and from the above experimental results, it clearly evident that SULPG with low computational cost than other descriptors, has shown high discriminative power and excellent robustness to various variations of human faces i.e. pose, expression, moderate illumination variation, accessory and lighting. The performance of the SULPG is not affected wherever the images exhibits high illumination and blur variations. This is mainly to gradient strategy on SULPG images. The SULPG is experimented on large size databases and the descriptor extracted more discriminative information in the form of stable transitions, which are subsets of fundamental units of texture. The excellent performance of SULPG can be explained as follows. It encodes the stable fundamental information of facial images while treating non-significant and noisy information of facial images under one label and the gradient strategy ensures good complementarily in dealing with illumination and blur variations.

\section{Conclusions}

In this paper we presented SULPG, a novel feature extraction method for Face identification, based on the impressive results of visual perception researches [33-34]. After an in-depth study, on the descriptors based on LBP in various classifications, recognition analysis issues, the present paper derived a new variant of LBP called SULPG with limited, consistent and stable features for face representation. The SULPG represents significant texture features like lines, curves, edges, holes, blobs etc... The SULPG descriptor is exploited on two gradient images i.e. on $\mathrm{x}$ and $\mathrm{y}$ axis of the raw image to generate SULPG image. The enhanced SULPG contains more discriminative information and it overcomes the illumination effect. The proposed SULPG descriptor is compared with other existing descriptors in a comprehensive and systematic manner and tested on five large facial databases. The method achieves stable and high performance over the other descriptors with respect to various challenges such as illumination, occlusion, facial expressions, pose variations and time-lapse images, by reducing the number of image features by reducing the overall dimensionality.

\section{References}

[1] S. Liao, A. K. Jain, and S. Z. Li, "Partial face recognition: Alignment free approach," IEEE Trans. Pattern Anal. Mach. Intell., vol. 35, no. 5, pp. 1193-1205, May 2013. https://doi.org/10.1109/TPAMI.2012.191.

[2] C. Ding, C. Xu, and D. Tao, "Multi-task pose-invariant face recognition," IEEE Trans. Image Process., vol. 24, no. 3, pp. 980-993, Mar. 2015. https://doi.org/10.1109/TIP.2015.2390959.

[3] R. Ibrahim and Z. M. Zin, "Study of automated face recognition system for of_ce door access control application," in Proc. IEEE Int Conf. Commun. Softw. Netw. May 2011, pp. 132-136.

[4] W. Chongwen, L. Huan, and M. Ming, "Face recognition technology based on identication card," in Proc. Int. Conf. Intell. Comput. Technol. Autom., 2012, pp. 173-176.

[5] V. K. N. Kumar and B. Srinivasan, "Enhancement of security and privacy in biometric passport inspection system using face, Finger- print, and iris recognition," Int. J. Comput. Netw. Inf. Secur., vol. 4, no. 8, pp. 55-64, Aug. 2012.

[6] M. Zhou, L. Liang, J. Sun, and Y. Wang, "AAM based face tracking with temporal matching and face segmentation," in Proc. IEEE Comput. Soc. Conf. Comput. Vis. Pattern Recognit., Jun. 2010, pp. 701_708. https://doi.org/10.1109/CVPR.2010.5540146.

[7] Y. Zhang, W. Lin, B. Zhou, Z. Chen, B. Sheng, and J. Wu, 'Facial expression cloning with elastic and muscle models," J. Vis. Commun. Image Represent, vol. 25, no. 5, pp. 916_927, Jul. 2014.

[8] I. Naseem, R. Togneri, and M. Bennamoun, "Linear regression for face recognition," IEEE Trans. Pattern Anal. Mach. Intell., vol. 32, no. 11, pp. 2106-2112, Nov. 2010 https://doi.org/10.1109/TPAMI.2010.128.

[9] E. Elhamifar and R. Vidal, "Block-sparse recovery via convex optimization," IEEE Trans. Signal Process. vol. 60, no. 8, pp. 4094 4107, Aug. 2012. https://doi.org/10.1109/TSP.2012.2196694.

[10] Y. Taigman, M. Yang, M. Ranzato, and L. Wolf, "DeepFace: Closingthe gap to human-level performance in face verification," in Proc. IEEE Conf. Comput. Vis. Pattern Recog., 2014, pp. 17011708. https://doi.org/10.1109/CVPR.2014.220.

[11] M. Turk and A. Pentland, "Eigenfaces for recognition," J. Cognit. Neurosci. vol. 3, no. 1, pp. 71-86, Jan. 1991. https://doi.org/10.1162/jocn.1991.3.1.71.

[12] Z. Cao, Q. Yin, X. Tang, and J. Sun, "Face recognition with learning-based descriptor," in Proc. Comput. Vis. Pattern Recog., 2010, pp. 2707-2714.

[13] C. Chan, J. Kittler, and K. Messer, "Multi-scale local binary pattern histograms for face recognition," in Proc. Int. Conf. Biometrics, 2007, pp. 809-818. https://doi.org/10.1007/978-3-540-74549-5 85.

[14] S. ulHussain and B. Triggs, "Visual recognition using local quantized patterns," in Proc. Eur. Conf. Comput. Vis., 2012, pp. 716 729.

[15] S. Xie, S. Shan, X. Chen, and J. Chen, "Fusing local patterns ofGabor magnitude and phase for face recognition," IEEE Trans.Image Process., vol. 19, no. 5, pp. 1349-1361, May 2010. https://doi.org/10.1109/TIP.2010.2041397.

[16] Wang X, ZhangYMuX et al (2012) the face recognition algorithm based on improved LBP. OptoElectronEng 7:76-80.

[17] Cament LA, Galdames FJ, Bowyer KW et al (2015) Face recognition under pose variation with localGabor features enhanced by active shape and statistical models. Pattern Recognit 48(11):33713384. https://doi.org/10.1016/j.patcog.2015.05.017.

[18] T. Ojala, M. Pietik"ainen, and T. Maenpaa, "Multi-resolution grayscale and rotation invariant texture classification with local binary patterns," IEEE Trans. Pattern Anal. Mach. Intell., vol. 24, no. 7, pp. 971-987, Jul. 2002 https://doi.org/10.1109/TPAMI.2002.1017623.

[19] T. Ojala, M. Pietik"ainen, and D. Harwood, "A comparative study of texture measures with classification based on featured distribution," Pattern Recog., vol. 29, no. 1, pp. 51-59, 1996. https://doi.org/10.1016/0031-3203(95)00067-4.

[20] T. Ahonen, A. Hadid, and M. Pietik"ainen, "Face description with local binary patterns: Application to face recognition," IEEE Trans. Pattern Anal. Mach. Intell., vol. 28, no. 12, pp. 2037-2041, Dec. 2006. https://doi.org/10.1109/TPAMI.2006.244.

[21] X. Tan and B. Triggs, "Enhanced local texture feature sets for facerecognition under difficult lighting conditions," in Proc. Anal. Model. Faces Gestures, 2007, pp. 168-182. https://doi.org/10.1007/978-3-540-76390-1_66.

[22] S. Liao and A. C. S. Chung, "Face recognition by using elongated local binary patterns with average maximum distance gradient magnitude," in Proc. Asian Conf. Comput. Vis., 2007, pp. 672-679.

[23] L. Zhang, R. Chu, S. Xiang, and S. Z. Li, "Face detection based onMulti-Block LBP representation," in Proc. Int. Conf. Biometrics, 2007, pp. 11-18.

[24] C. Shan, S. Gong, and P. W. McOwan, "Facial expression recognition based on local binary patterns: A comprehensive study," Image Vis. Comput., vol. 27, no. 6, pp. 803-816, May 2009. https://doi.org/10.1016/j.imavis.2008.08.005.

[25] T. Gritti, C. Shan, V. Jeanne, and R. Braspenning, "Local features based facial expression recognition with face registration errors," presented at the IEEE Int. Conf. Autom. Face Gesture Recog, Amsterdam, The Netherlands, Sep. 2008 https://doi.org/10.1109/AFGR.2008.4813379

[26] Z. Yang and H. Ai, "Demographic classification with local binary patterns," in Proc. Int. Conf. Biometrics, 2007, pp. 464-473. https://doi.org/10.1007/978-3-540-74549-5_49.

[27] J. Trefny, J. Matas (2010) Extended set of local binary pattern for rapid object detection, in: Proceedings of Computer Vision Winter Workshop. 
[28] LinlinShen $Æ$ Li Bai, A review on Gabor wavelets for face recognition, Pattern Anal Applic (2006) 9:273-292. https://doi.org/10.1007/s10044-006-0033-y.

[29] T. Ahonen, E. Rahtu, V. Ojansivu, and J. Heikkila, "Recognition ofblurred faces using local phase quantization," in Proc. Int Conf.Pattern Recog., 2008, pp. 1-4.

[30] N.-S. Vu and A. Caplier, "Enhanced patterns of oriented edgemagnitudes for face recognition and image matching," IEEETrans. Image Process. vol. 21, no. 3, pp. 1352-1365, Mar. 2012. https://doi.org/10.1109/TIP.2011.2166974

[31] T. Ahonen, A. Hadid, and M. Pietikäinen, "Face description with local binary patterns: Application to face recognition," IEEE Trans. Pattern Anal. Mach. Intell., vol. 28, no. 12, pp. 2037-2041, Dec. 2006 https://doi.org/10.1109/TPAMI.2006.244.

[32] E. H. Land and J. J. McCann, "Lightness and retinex theory," J. Opt. Soc. Amer., vol. 61, no. 1, pp. 1-11, 1971 https://doi.org/10.1364/JOSA.61.000001.

[33] B. Julesz, "Textons, the elements of texture perception, and theirinteractions," Nature, vol. 290, no. 5802, pp. 91-97, Mar. 1981 https://doi.org/10.1038/290091a0.

[34] V. Vijaya Kumar, K. Srinivasa Reddy, V. Venkata Krishna "Face Recognition Using Prominent LBP Model”, International Journal of Applied Engineering Research, Vol. 10, Iss. 2, 2015, pp. 4373-4384, ISSN: 0973-4562

[35] J. Trefny and J. Matas, "Extended set of local binary patterns, for rapid object detection," in Proc. Comput. Vis. Winter Workshop, 2010.

[36] P. Phillips, H. Moon, S. Rizvi, and P. Rauss, "The FERET evaluation methodology for face-recognition algorithms," IEEE Trans. Pattern Anal. Mach. Intell., vol. 22, no. 10, pp. 1090-1104, Oct. 2000. https://doi.org/10.1109/34.879790.

[37] Wen Gao, Bo Cao, Shiguang Shan, Xilin Chen, Delong Zhou, Xiaohua Zhang, Debin Zhao. The CAS-PEAL Large-Scale Chinese Face Database and Baseline Evaluations. IEEE Trans. on System Man, and Cybernetics (Part A), vol.38, no.1, pp149-161. 2008.1

[38] G. B. Huang, M. Ramesh, T. Berg, and E. Learned-Miller, "Labeled faces in the wild: A database for studying face recognition in unconstrained environments," Dept. Comput. Sci., Univ. Massachusetts, Amherst, MA, USA, Tech. Rep. 07-49, Oct. 2007.

[39] A. Georghiades, P. Belhumeur, and D. Kriegman, "From few to many: Illumination cone models for face recognition under variable lighting and pose," IEEE Trans. Pattern Anal. Mach. Intell., vol. 23, no. 6, pp. 643-660, Jun. 2001 https://doi.org/10.1109/34.927464.

[40] Samaria, Ferdinando S., and Andy C. Harter. "Parameterisation of a stochastic model for human face identification." In Applications ofComputer Vision, 1994, Proceedings of the Second IEEE Workshop on, pp. 138-142. IEEE, 1994

[41] N.-S. Vu and A. Caplier, "Face recognition with patterns of oriented edge magnitudes," in Proc. ECCV, 2010, pp. 313-326. https://doi.org/10.1007/978-3-642-15549-9_23.

[42] J. G. Daugman, "Uncertainty relation for resolution in space, spatialfrequency, and orientation optimized by two-dimensional visual cortical filters," J. Opt. Soc. Amer. A, Opt., Image Sci., Vis., vol. 2, no. 7, pp. 1160-1169, 1985. https://doi.org/10.1364/JOSAA.2.001160. 